\title{
Point contact studies of the superconducting gap of $\mathrm{CeRu}_{2}$
}

\author{
Yu. G. Naidyuk, A. V. Moskalenko, and I. K. Yanson, \\ B. Verkin Institute for Low Temperature Physics and Engineering, National Academy of Sciences of Ukraine, \\ 47, Lenin Ave., 310164, Kharkov, Ukraine \\ C. Geibel
}

Institut für Festkörperphysik, TU Darmstadt , D-64289 Darmstadt, Germany

Received December 25, 1997.

\begin{abstract}
The measured $d V / d I(V)$ curves of point contacts between the intermetallic compound $\mathrm{CeRu}_{2}$ in the superconducting state and the normal metal $\mathrm{Cu}$ correspond fairly well to the Blonder-Tinkham -Klapwijk model. It is used to obtain temperature and magnetic field dependence of the superconducting gap $\Delta$. The $\Delta(T)$ dependence corresponds well to the BCS theory with $2 \Delta(0) / k_{B} T_{c}^{*}=3.1 \pm 0.1$. Additionally, a region of gapless superconductivity between $T_{c}^{*} \simeq 4.4-5.4 \mathrm{~K}$ and $T_{c}^{\mathrm{bulk}} \simeq 6.2 \mathrm{~K}$ is found. The gap decreases approximately linearly in a magnetic field and vanishes in a field $B_{c}^{*} \simeq 3.5 \mathrm{~T}$, which is well below the upper critical field $B_{c 2} \simeq 6 \mathrm{~T}$ and close to the irreversibility field.

PACS: 74.80.Fp, 74.70.Tx, 73.40.Jn
\end{abstract}

\section{Introduction}

The recently renewed interest in the intermetallic compound $\mathrm{CeRu}_{2}$ is attributable to its anomalous magnetic and transport properties in the superconducting (SC) mixed state. As one of the possible reasons for this the generalized Fulde-FerrellLarkin-Ovchinnikov state [1] is discussed with a spatially modulated SC order parameter (OP), which could have planar nodes aligned perpendicularly to the vortices. Until now, however, only a few direct investigations of the OP have been reported. From Shottky tunneling experiments [2] the SC gap was estimated to be $2 \Delta(0) / k_{B} T_{c}=$ 11-12. Subsequent attempts by using metallic point contacts (PC) [3] revealed a rather low value (less than 2). Recent break-junction tunneling measurements [4] yielded $2 \Delta(0) / k_{B} T_{c}=4.2$, quite different from the former results, indicating specific difficulties of the aforementioned methods. In the latter investigation a BCS-type temperature dependence of the gap peculiarities was ascertained. Unfortunately, no magnetic field behavior of SC gap features was established in any of the studies, although magnetic field is one of the sensitive probes of superconductivity.
The aim of this paper is to study the temperature and the magnetic-field dependences of the SC gap in $\mathrm{CeRu}_{2}$ using a metallic PC between the $\mathrm{CeRu}_{2}$ and the normal metal. We obtained $d V / d I(V)$ characteristics, which correspond well to the Blonder-Tinkham-Klapwijk (BTK) model [5], taking into account the well-known mechanism of the Andreev reflection (AR) at a normal metal-superconductor $(N-S)$ interface. The gap value was determined directly from a fit according to this BTK model.

\section{Experiment and results}

We used $\mathrm{CeRu}_{2}$ polycrystals with $R R R \simeq 14$ and $T_{c}=6.2 \mathrm{~K}$. The PC's were prepared by touching the $\mathrm{CeRu}_{2}$ crystal with the edge of a Cu electrode. The freshly broken $\mathrm{CeRu}_{2}$ surface gave the best $d V / d I$ curves with a pronounced minimum (or double minimum). The sample was immersed in liquid ${ }^{4} \mathrm{He}$ to ensure good thermal coupling. The differential resistance $d V / d I$ was recorded vs. bias voltage $V$ using a standard lock-in amplifier technique, modulating the direct current $I$ with a small ac component. The applied magnetic field $B$ was approximately parallel to the contact axis. 


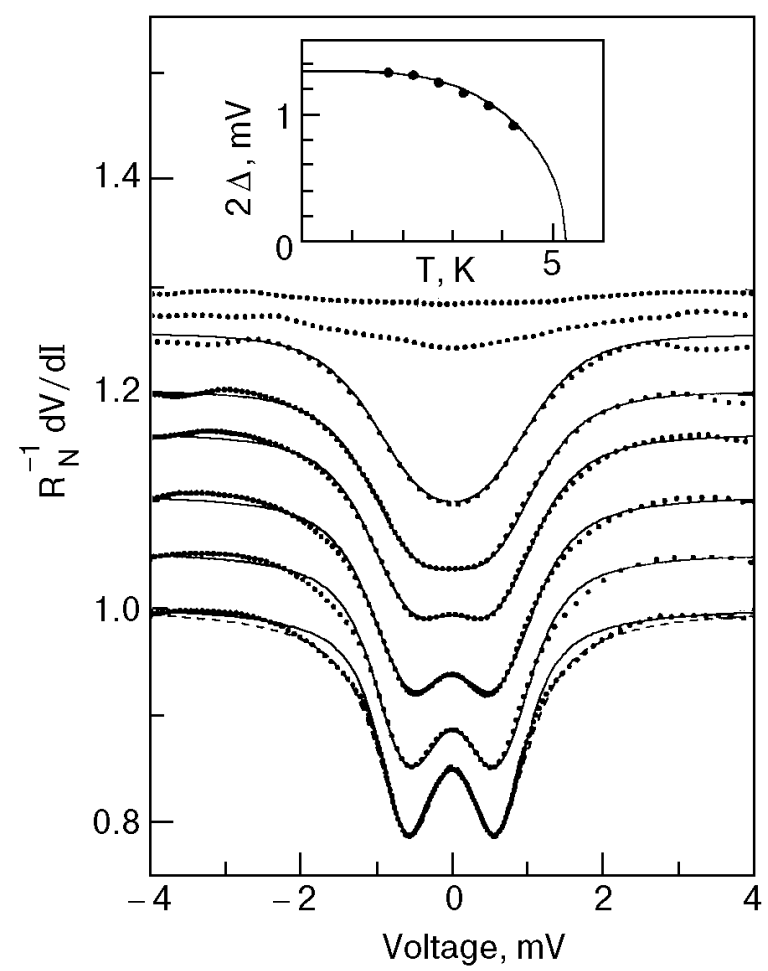

Fig. 1. The temperature dependence of the reduced $d V / d I$ curves vs. bias $V$ (points) for a $\mathrm{CeRu}_{2}-\mathrm{Cu}$ contact with $R_{N}=1.4 \Omega$ along with a fit using Eq. (1) with $\Gamma=0$ (solid lines) and $\Gamma / \Delta=0.25$ (dashed line). The left scale corresponds to the experimental curves and to the dashed line. The curves are offset vertically for clarity. The temperatures from top curve to bottom curve $\mathrm{T}, \mathrm{K}: 6.2,5.2,4.2,3.7,3.2,2.7,2.2$, and $1.7 \mathrm{~K}$. Inset: reduced gap $\Delta$ vs. temperature from the fit with $\Gamma=0$. The solid line is BCS curve with $2 \Delta(0) / k_{B} T_{c}^{*} \simeq 3$.

Figures 1 and 2 show the $d V / d I$ curves vs. $V$ of the $\mathrm{CeRu}_{2}-\mathrm{Cu}$ contacts at various temperatures and magnetic fields. The double-minimum structure of about $V=0$ is clearly seen. This feature is a strong indicator of the Andreev reflection in the presence of weak quasiparticle reflection at the $N-S$ interface, as described by the phenomenological barrier strength parameter $Z$ [5]. The $I-V$ characteristic in this case is

$$
\begin{gathered}
I(V) \sim \int_{-\infty}^{\infty} T(E)[f(E-e V)-f(E)] d E, \\
T(E)=\frac{2 \Delta^{2}}{E^{2}+\left(\Delta^{2}-E^{2}\right)\left(2 Z^{2}+1\right)^{2}}, \quad|E|<\Delta, \\
T(E)=\frac{2|E|}{|E|+\left(E^{2}-\Delta^{2}\right)^{1 / 2}\left(2 Z^{2}+1\right)},|E|>\Delta,
\end{gathered}
$$

where $f(E)$ is the Fermi distribution function and $\Delta$ is the superconducting gap. In the modified BTK theory the broadening of the quasiparticle density

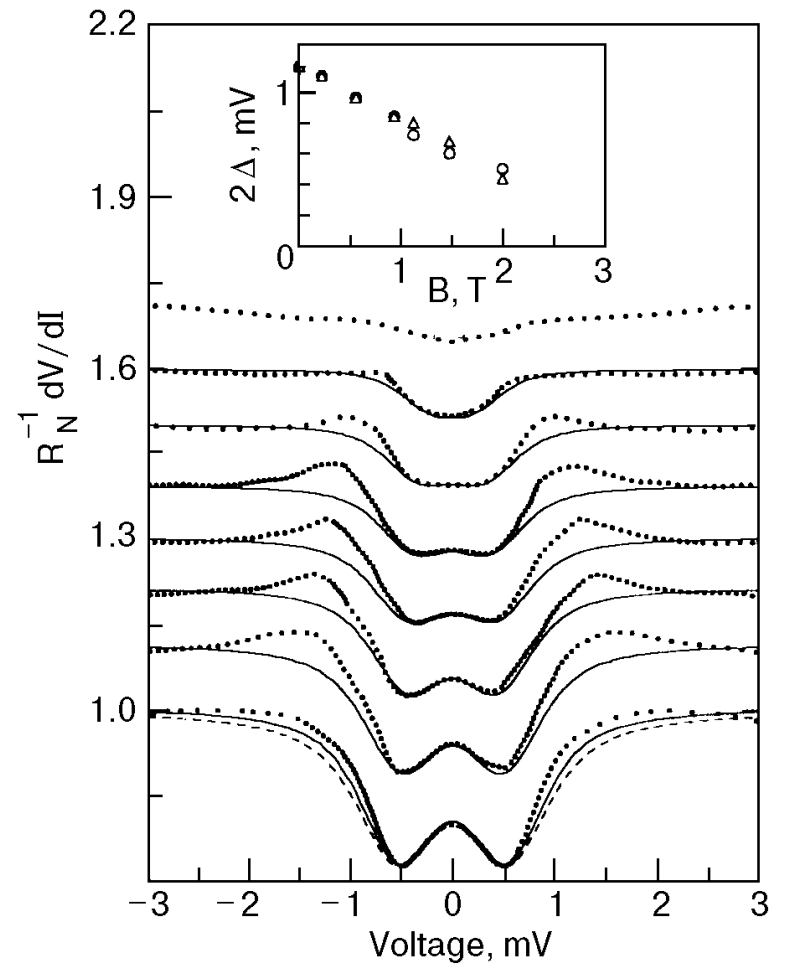

Fig. 2. The magnetic field dependence of the reduced $d V / d I$ curves vs. bias $V$ (points) for $\mathrm{CeRu}_{2}$-Cu contact at $T=1.8 \mathrm{~K}$ with $R_{N}=1.1 \Omega$ along with the fit using Eq. (1) with $\Gamma=0$ (solid lines) and $\Gamma / \Delta=0.1$ (dashed line). The left scale corresponds to the experimental curves and to the dashed line. The curves are offset vertically for clarity. The magnetic fields from top curve to bottom curve B, T: $2.8,2,1.5,1.13,0.94,0.56$, 0.22 , and 0 . Inset: $2 \Delta$ vs. magnetic field using the fit with $\Gamma=0$ (triangles) and $\Gamma \neq 0$ (circles).

of states $N(E, \Gamma)$ in the superconductor for the finite quasiparticle lifetime $\tau$ is taken into account. According to the Dynes et al. [6],

$$
N(E, \Gamma)=\operatorname{Re}\left\{\frac{E-i \Gamma}{\left[(E-i \Gamma)^{2}-\Delta^{2}\right]^{1 / 2}}\right\},
$$

where the broadening parameter is $\Gamma=\hbar / \tau$.

In order to obtain the temperature and magnetic field dependences of $\Delta$ we fitted the measured $d V / d I$ curves according to Eq. (1) for $\Gamma=0$ and $\Gamma \neq 0$. The parameter $Z$ was kept fixed, independent of the temperature and magnetic field. The plots of $\Delta(T)$ and $\Delta(B)$ of different $\mathrm{CeRu}_{2}$ contacts are shown in the insets in Figs. 1-3. The original BTK theory, i.e., with $\Gamma=0$, usually fitted better the behavior of $d V / d I$ near the double-minimum (see Figs. 1 and 2), especially compared to the fit with large $\Gamma / \Delta>0.1$ ratio, but it does not reproduce the relative change of $d V / d I$ between $V=0$ and large biases. On the average, we obtained a reduced gap of $2 \Delta(0) / k_{B} T_{c}^{*}=3.1 \pm 0.1$. Using the modified BTK fit, we see that the $2 \Delta(0) / k_{B} T_{c}^{*}$ ratio de- 

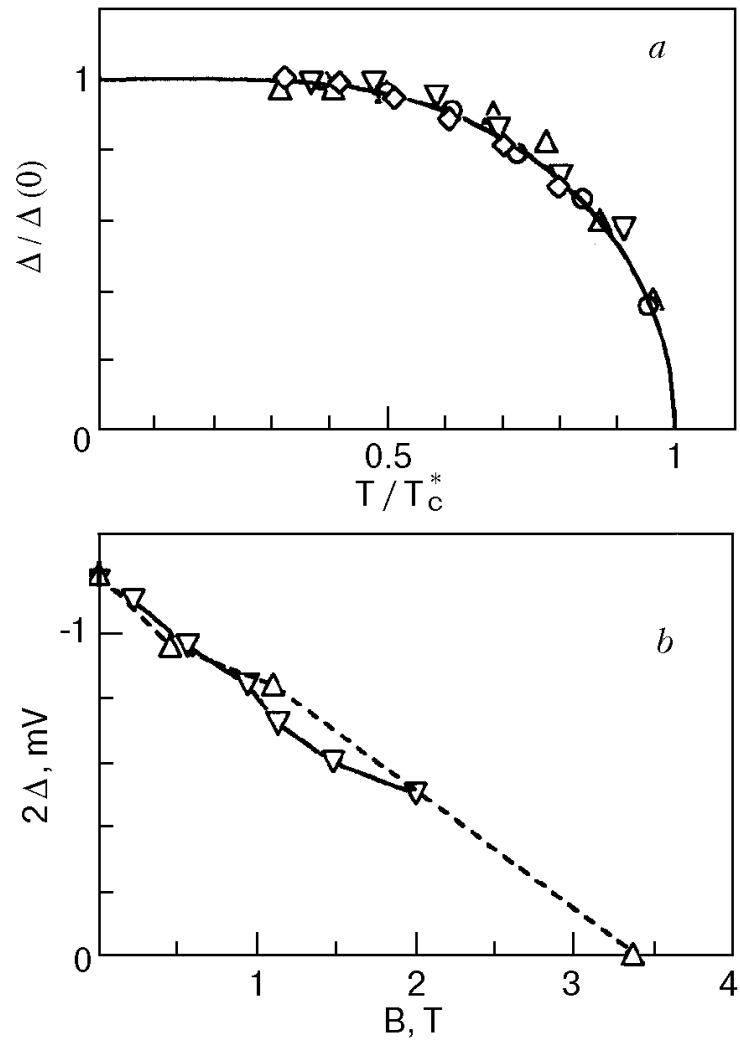

Fig. 3. a) Reduced $\Delta$ vs. temperature of different PC along with the BCS curve, b) $2 \Delta$ vs. magnetic field of different PC. The lines connect the symbols for clarity.

creases (by about 10-30\%) with increasing $\Gamma / \Delta$ value (correspondingly from 0.25 to 0.75 ).

As can be seen from Fig. 1, the $d V / d I$ curve at $T=5.2 \mathrm{~K}$ still exhibits a broad and shallow SC minimum, but it is not possible to fit it reasonably to any $\Delta$. Since for this contact BCS $\Delta(T)$ behavior (see the inset in Fig. 1) extrapolates to $T_{c}^{*} \simeq$ $\simeq 5.25 \mathrm{~K}$, we conclude that the small SC features above $T_{c}^{*}$ and below $T_{c}^{\text {bulk }}=6.2 \mathrm{~K}$ correspond to a gapless state. For different contacts $T_{c}^{*}$ is between 4.4 and $5.25 \mathrm{~K}$ probably because of the local superconducting properties in the contact region.

The magnetic field dependence $\Delta(B)$ is almost linear (see the insets in Figs. 2 and $3, b$ ), with a critical field $B_{c}^{*}$ about $3.4 \mathrm{~T}$. This is quite different to what is expected for a type-II superconductor [7].

\section{Discussion and conclusions}

The other possibility of yielding a maximum at $\mathrm{V}=0$ and similar double-minimum structure on $d V / d I$ is Kondo scattering superimposition with SC minimum, which was recently discussed in detail in Ref. 8. However, in a magnetic field this maximum vanishes (see Fig. 2) without the splitting characteristic of the Kondo effect [9]. More- over, the fit according to BTK theory obeys the measured characteristics, quite well thus making the magnetic scattering unlikely to occur in the observed structure.

According to our findings, $\mathrm{CeRu}_{2}$ is a BCS-like superconductor. The relatively small $2 \Delta(0) / k_{B} T_{c}^{*}$ value (corresponding to weak-coupling superconductivity) and the region of gapless superconductivity close to $T_{c}^{\text {bulk }}$ are possibly related to the pair-breaking effect caused, e.g., by the Ce local magnetic moments which are distributed randomly in the contact region. This pair-breaking effect may also contribute to the small value of the reduced gap [10]. For the reason mentioned above it seems reasonable to include Kondo scattering in the BTK theory for a better understanding of the point contact characteristics and the relationship between superconductivity and magnetism in the point contacts.

The gradually decreasing gap in the magnetic field makes the transition to the Fulde-FerrellLarkin-Ovchinnikov state unlikely, which should be of the first order. The linear decrease of $\Delta(B)$ and the low magnetic field at which the superconducting gap vanishes remains an enigma. The close coincidence of $B^{*}$ with the irreversibility field [11] points to a change of the vortex dynamics as a possible reason. To solve these puzzles it is necessary to carry out further experiments on the temperature and magnetic field $d V / d I(V)$ dependences of the same point contact much closer to $T_{c}^{*}$ and $B_{c}^{*}$ using a more perfect $\mathrm{CeRu}_{2}$ single crystal.

This work was carried out, in part, thanks to the support of A. V. Humboldt Stiftung. We are indebted to K. Gloos for a useful discussion, R. Häussler for BTK fit program, and R. Clemens for preparing the samples. I. K. Yanson acknowledges the financial support under INTAS Project No.94-3562.

1. M. Tachiki et al., Z. Phys. B100, 369 (1996).

2. W. Schmitt and G. Guntherodt, J. Magn. Magn. Mater. 47-48, 542 (1985).

3. M. E. Solanki-Moser, Ph. D. Thesis, ETH Zürich (1987).

4. T. Ekino, H. Fujii, T. Nakama, and K. Yagasaki, Czech. J. Phys. 46, 783 (1996); Phys. Rev. 56, 7851 (1997).

5. G. E. Blonder, M. Tinkham, and T. M. Klapwijk, Phys. Rev. 25, 4515 (1982).

6. R. C. Dynes, V. Narayanamurti, and J. P. Garno, Phys. Rev. Lett. 21, 1509 (1978).

7. K. Maki, in: Superconductivity, R. D. Parks (ed.) Marcel Dekker, New York (1969).

8. Yu. G. Naidyuk, K. Gloos, and A. A. Menovsky, J. Phys.: Condens. Matter 9, 6279 (1997).

9. A. G. M. Jansen, A. P. van Gelder, and P. Wyder, J. Phys. C13, 6073 (1980).

10. M. Tinkham, Introduction to Superconductivity, McGrawHill, New York (1980).

11. A. D. Huxley et al., J. Phys.: Condens. Matter 5, 7709 (1993). 